\title{
Oclusão Percutânea de Comunicação Interventricular por Via Arterial: Técnica Alternativa
}

\author{
Francisco José Araujo Chamié de Queiroz¹, Luiz Carlos do Nascimento Simões², \\ Daniel Silva Chamié de Queiroz ${ }^{2}$, João Carlos Tress², Maximiliano Otero Lacoste ${ }^{2}$
}

\section{RESUMO}

Introdução: A oclusão percutânea das comunicações interventriculares (CIVs) surgiu como uma alternativa ao tratamento cirúrgico ainda na década de 1980. Durante o implante da prótese por via anterógrada, a formação de uma alça arteriovenosa com a guia é tecnicamente difícil e demanda tempo. O objetivo deste estudo foi apresentar a experiência dos autores com a via retrógada e mostrar sua exequibilidade nos diversos tipos de CIV. Métodos: Foram incluídos pacientes portadores de defeitos do septo interventricular, que não apresentassem outros defeitos associados. Os casos foram selecionados por meio de ecocardiografia transtorácica. Resultados: No total, 8 pacientes foram submetidos ao procedimento, 4 portadores de CIV muscular e 4 portadores de CIV perimembranosa. Metade era do sexo masculino, as idades variaram de 7 anos a 32 anos $(18,6 \pm 7,8$ anos) e os pesos, de $30 \mathrm{~kg}$ a $97 \mathrm{~kg}(66,12 \pm 24,7 \mathrm{~kg})$. Os diâmetros dos defeitos variaram de $2 \mathrm{~mm}$ a $8,6 \mathrm{~mm}(5 \pm$ 2,4 mm). O implante foi possível em todos os casos, e a prótese mais utilizada foi a Lifetech Cera ${ }^{\mathrm{TM}}$ tipo I. As próteses Amplatzer ${ }^{\mathrm{TM}}$ Muscular VSD Occluder e Lifetech Cera ${ }^{\mathrm{TM}}$ tipo II também foram empregadas. Duas próteses embolizaram imediatamente após o implante e foram retiradas. Oclusão imediata ocorreu em $75 \%$. Uma paciente apresentou bloqueio atrioventricular total na semana seguinte ao procedimento. Foi tratada com esteroides, obtendo a reversão para ritmo sinusal. Conclusões: A utilização da via retrógrada mostrou-se eficaz na oclusão de todos os tipos morfológicos de CIV com próteses de duplo disco. Os autores sugerem essa técnica como alternativa para a oclusão percutânea das CIVs de qualquer morfologia.

DESCRITORES: Comunicação interventricular. Cateterismo cardíaco. Próteses e implantes. Cardiopatias congênitas.

\footnotetext{
Setor de Hemodinâmica Pediátrica do Hospital dos Servidores do Estado - MS-RJ - Rio de Janeiro, RJ, Brasil.

2 INTERCAT - Cardiologia Intervencionista - Rio de Janeiro, RJ, Brasil. Correspondência: Francisco José Araujo Chamié de Queiroz. Av. Borges de Medeiros, 3.501/103 - Lagoa - Rio de Janeiro, RJ, Brasil CEP 22470-001

E-mail: fchamie@pobox.com

Recebido em: 4/7/2011 • Aceito em: 3/9/2011
}

\section{ABSTRACT}

Retrograde Transcatheter Occlusion of Ventricular Septal Defects: An Alternative Technique

Background: Transcatheter occlusion of ventricular septal defects (VSDs) was developed as an alternative to surgical treatment in the $80^{\prime}$ s. During anterograde device implantation, the formation of an arteriovenous loop with a guidewire is technically difficult and time consuming. The aim of this study is to present the authors' experience with the retrograde approach and demonstrate its feasibility in different types of VSD. Methods: Patients with VSD with no other associated defects were included in the study. Cases were selected by transthoracic echocardiograms. Results: A total of eight patients were submitted to the procedure, 4 with muscular VSD and 4 with perimembranous VSD. Half of them were male, with ages ranging from 7 to 32 years $(18.6$ \pm 7.8 years $)$ and weight ranging from 30 to $97 \mathrm{~kg}(66.12+$ $24.7 \mathrm{~kg})$. The defect diameter ranged from 2 to $8.6 \mathrm{~mm}(5$ $\pm 2.4 \mathrm{~mm}$ ). Implantation was possible in all patients and the most commonly used device was Lifetech Cera ${ }^{\mathrm{TM}}$ Type I. Amplatzer $^{\mathrm{TM}}$ Muscular VSD Occluder, and Lifetech Cera ${ }^{\mathrm{TM}}$ Type II devices were also used. Two devices embolized immediately after implantation and were snared out. There was immediate occlusion in $75 \%$ of the cases. One patient had a complete atrioventricular block in the week following the procedure. The patient was treated with steroids, and was reverted to normal sinus rhythm. Conclusions: Retrograde transcatheter VSD occlusion with double disk devices proved to be effective in different types of VSD. The authors suggest this technique as an alternative to percutaneous occlusion of all types of VSD.

KEY-WORDS: Heart septal defects, ventricular. Heart catheterization. Prostheses and implants. Heart defects, congenital.

A s comunicações interventriculares (CIVs) são frequentes, representando $25 \%$ a $30 \%$ de todos os defeitos congênitos do coração ${ }^{1}$ A cirurgia convencional apresenta baixa mortalidade, mas pode estar associada a morbidade significativa (arritmias, síndrome pós-pericardiotomia, infecções pulmonares e mediastinais), internação prolongada, cicatriz da esternotomia e desconforto para o paciente. ${ }^{2,3}$ 
A oclusão percutânea das CIVs surgiu como uma alternativa ao tratamento cirúrgico, ainda na década de $1980 .{ }^{4}$ Os resultados iniciais eram heterogêneos, uma vez que os dispositivos utilizados não eram desenhados especificamente para essa finalidade. ${ }^{4-7}$ Foi apenas com o lançamento da prótese Amplatzer ${ }^{\mathrm{TM}}$ (AGA, Golden Valley, Estados Unidos), para CIV muscular, que a técnica ganhou maior aceitação. ${ }^{8}$ Vários trabalhos se seguiram, relatando considerações técnicas e resultados iniciais e tardios. ${ }^{9-13}$ A técnica utilizada na maioria dos pacientes consistia em cruzar o defeito pelo ventrículo esquerdo com uma guia de troca, que era laçada nas cavidades direitas e retirada pela veia femoral ou jugular, criando uma alça arteriovenosa. A bainha longa, com a prótese carregada em seu interior, era introduzida pela veia (abordagem anterógrada) e avançada até o lado esquerdo do septo interventricular, onde se iniciava o implante. Essa técnica acarretava maior dificuldade e aumentava o tempo do procedimento, com consequente aumento da exposição aos raios $X$ para paciente $\mathrm{e}$ equipe.

Alguns autores propuseram o implante pelo lado arterial (abordagem retrógrada) quando a técnica habitual falhava..$^{9,10,13-16} \mathrm{Em} \mathrm{2006}$, Jameel et al. ${ }^{17}$ relataram o fechamento retrógrado como opção primária, com sucesso, em 7 pacientes. Posteriormente um dispositivo especificamente desenhado para a oclusão dos defeitos perimembranosos (CIVs perimembranosas), Amplatzer ${ }^{\text {TM }}$ Membranous VSD Occluder (Aga Medical, Golden Valley, Estados Unidos) ${ }^{18,19}$, tornou-se disponível e trabalhos atestando sua segurança e eficácia, utilizando a técnica anterógrada, foram publicados. ${ }^{19-24}$ $\mathrm{O}$ advento de dispositivos de malha de nitinol de duplo disco, desenhados especificamente para a oclusão de CIVs perimembranosas, possibilitou o fechamento retrógrado desse tipo morfológico de defeito. ${ }^{24,25}$

O objetivo deste estudo foi apresentar a experiência dos autores com a via retrógada e mostrar sua exequibilidade na oclusão dos diversos tipos de CIV.

\section{MÉTODOS}

\section{Desenho do estudo e seleção dos pacientes}

Este é um estudo prospectivo, unicêntrico, de braço único, que visa a apresentar a experiência inicial dos autores com uma variação da técnica utilizada para oclusão percutânea das CIVs.

Para a aplicação dessa técnica foram avaliados, consecutivamente, todos os pacientes portadores de CIVs congênitas encaminhados à INTERCAT - Cardiologia Intervencionista (Rio de Janeiro, RJ). Foram incluídos pacientes sem restrição de idade, com peso $>12 \mathrm{~kg}$, portadores de defeitos localizados em qualquer ponto do septo interventricular passíveis de serem fechados com próteses, e que não apresentassem outros defeitos com indicação de correção cirúrgica.

Os casos foram selecionados por meio de ecocardiografia transtorácica (ETT) com mapeamento de fluxo em cores. As dimensões do defeito não constituíram critério de exclusão.

Foram utilizadas as seguintes próteses:

- Amplatzer ${ }^{\mathrm{TM}}$ Muscular VSD Occluder (Aga Medical, Golden Valley, Estados Unidos) (Figura 1A) Prótese autoexpansível, constituída de dois discos simétricos de nitinol (liga de níquel e titânio), com poliéster em seu interior. $\mathrm{O}$ disco proximal tem uma rosca fêmea para conexão com o sistema de entrega. Ambos os discos são $4 \mathrm{~mm}$ maiores que a porção central (cintura), que mede de $4 \mathrm{~mm}$ a $18 \mathrm{~mm}$ de diâmetro, em incrementos de $2 \mathrm{~mm}$, e comprimento de $7 \mathrm{~mm}$. O sistema de entrega é composto de uma bainha longa, flexível, de $5 \mathrm{~F}$ a $9 \mathrm{~F}$, um carregador curto de calibre compatível, uma válvula hemostática em "Y", e um cabo metálico rosqueado na extremidade distal.

- Lifetech CeraTM Membranous VSD Occluder (Symmetric) tipo I (Lifetech Scientific, Shenzhen, China) (Figura 1B) - Prótese autoexpansível constituída de dois discos simétricos de nitinol, revestidos de cerâmica.
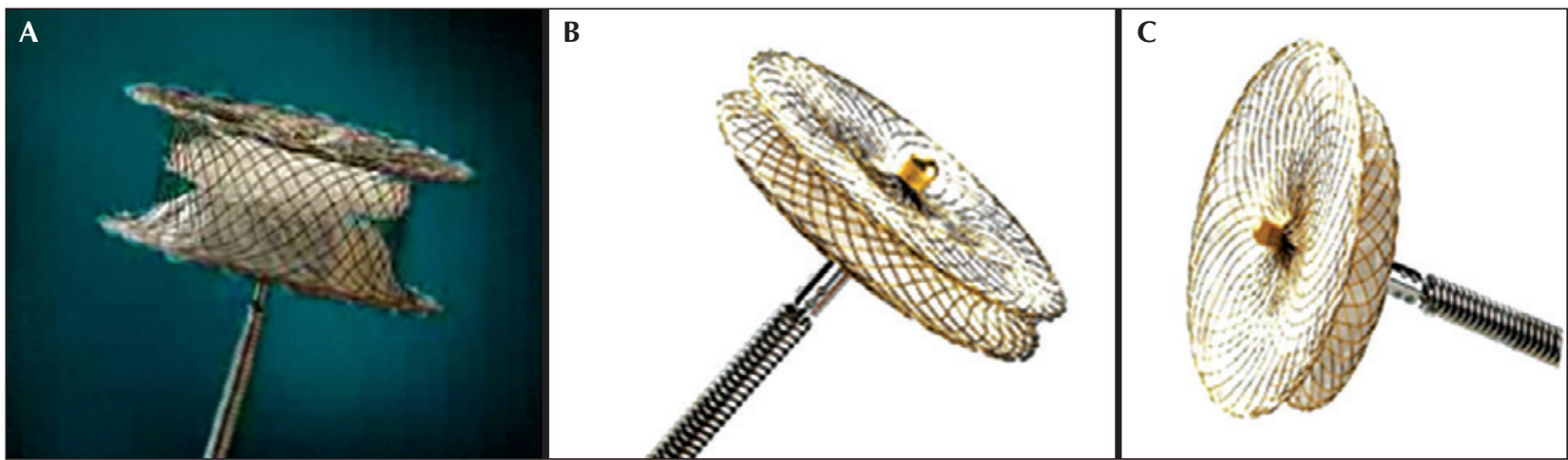

Figura 1 - Três tipos de prótese de duplo disco utilizadas. Em A, Amplatzer ${ }^{\text {TM }}$ Muscular VSD Occluder. Em B, Lifetech Cera ${ }^{\text {TM }}$ tipo I. Em C, Lifetech Cera $^{\mathrm{TM}}$ tipo II. 
O disco proximal tem uma rosca fêmea para conexão com o sistema de entrega. Os discos são $6 \mathrm{~mm}$ maiores que a porção central (cintura), que mede de $4 \mathrm{~mm}$ a $24 \mathrm{~mm}$, com incrementos de $1 \mathrm{~mm}$ até a cintura de $8 \mathrm{~mm}$, e incrementos de $2 \mathrm{~mm}$ para cinturas maiores, e tem $3 \mathrm{~mm}$ de comprimento. O sistema de entrega é composto de bainha longa e flexível de $5 \mathrm{~F}$ a $12 \mathrm{~F}$, carregador curto de tamanho compatível, válvula hemostática, e cabo metálico rosqueado na extremidade distal.

- Lifetech Cera ${ }^{\mathrm{TM}}$ Membranous VSD Occluder (Symmetric with larger left disk) tipo II (Lifetech Scientific, Shenzhen, China) (Figura 1C) - Prótese autoexpansível constituída de dois discos simétricos de nitinol, revestidos de cerâmica. O disco direito é $5 \mathrm{~mm}$ maior que a porção central (cintura) e o esquerdo é 2,6 mm maior que o direito, que tem uma rosca fêmea para conexão com o sistema de entrega. A porção central mede de $4 \mathrm{~mm}$ a $24 \mathrm{~mm}$, com incrementos de $1 \mathrm{~mm}$ até $8 \mathrm{~mm}$, e de $2 \mathrm{~mm}$ nos demais tamanhos, e tem $3 \mathrm{~mm}$ de comprimento. O sistema de entrega é composto de bainha longa e flexível de $5 \mathrm{~F}$ a $12 \mathrm{~F}$, carregador curto de tamanho compatível, válvula hemostática, e cabo metálico rosqueado na extremidade distal.

\section{Técnica}

Todos os procedimentos foram realizados sob anestesia geral com intubação orotraqueal. Ecocardiograma transesofágico (ETE) era realizado em seguida para confirmar o diagnóstico e obter mais detalhes anatômicos. Os procedimentos foram inteiramente monitorados pelo ETE, além da fluoroscopia. Profilaxia antimicrobiana com cefalotina intravenosa foi administrada na dose de $50 \mathrm{mg} / \mathrm{kg}$ (até um máximo de $2 \mathrm{~g}$ ) no momento do procedimento.

Foram obtidos acessos arterial e venoso por punção de veia e artéria femorais. Nos três primeiros casos foi obtido um segundo acesso arterial para auxiliar na visualização da prótese no momento do implante do dispositivo. Após a obtenção dos acessos vasculares, era administrada, por via endovenosa, heparina na dose de $100 \mathrm{UI} / \mathrm{kg}$ (até um máximo de $5.000 \mathrm{UI}$ ).

Todos os pacientes realizaram cateterismo cardíaco direito e esquerdo com registro de pressões em ventrículos e vasos da base. Foram obtidas angiografias em ventrículo esquerdo, nas incidências oblíqua anterior esquerda (OAE) em eixo longo (OAE 60 graus ou 70 graus, com inclinação cranial de 30 graus), nos casos de CIV perimembranosa e muscular, e oblíqua anterior direita 30 graus nos casos de CIV muscular infundibular. Foi realizada, também, aortografia panorâmica em OAE 30 graus para avaliar a competência valvar (Figura 2).

Depois da definição do tipo morfológico e da obtenção das medidas dos diâmetros de entrada do defeito no ventrículo esquerdo e saída no ventrículo direito, as CIVs foram cruzadas, retrogradamente. Uti- lizou-se um cateter de Judkins, pré-moldado para coronária direita (JR), com calibre de $4 \mathrm{~F}$ ou $5 \mathrm{~F}$, posicionado em frente à CIV, com uma guia hidrofílica 0,032 polegada/260 $\mathrm{cm}$ em seu interior. A guia era avançada, cruzando o orifício, e posicionada em cavidades direitas. Em seguida, o cateter era avançado sobre a guia e posicionado com o cuidado de manter sua extremidade livre no corpo do ventrículo direito e sem passar sob alguma parte do aparelho de sustentação da valva atrioventricular direita. Eventualmente, foi utilizada uma guia super-rígida (super stiff) 0,035 polegada $/ 260 \mathrm{~cm}$ posicionada, preferentemente, na artéria pulmonar esquerda para melhor suporte. Sobre ela era introduzida a bainha longa, de tamanho adequado ao tamanho da prótese escolhida, e posicionada no interior do ventrículo direito. Para confirmar a posição da bainha longa, era realizada injeção manual através do braço lateral da válvula hemostática em "Y".

A prótese escolhida era $1 \mathrm{~mm}$ a $2 \mathrm{~mm}$ maior que o menor orifício do defeito (em geral, o orifício de saída no ventrículo direito) e introduzida pelo interior da bainha até exteriorizar o disco distal. O sistema era, então, recuado até encontrar o septo interventricular. Nesse ponto, nova injeção manual era realizada para confirmar a posição do sistema e o disco proximal era exteriorizado no ventrículo esquerdo, recuando-se a bainha longa com a prótese mantida em posição. Nova injeção de teste, pela bainha longa ou por um cateter angiográfico auxiliar (pigtail), posicionado em ventrículo esquerdo, era realizada para confirmar a posição adequada da prótese, quando, então, o dispositivo era desenroscado, liberando-o do cabo de entrega (Figura 3). A realização de angiografias de controle em ventrículo esquerdo e aorta completava o procedimento de implante (Figura 4). Os introdutores eram retirados em sala e a hemostasia era obtida por compressão manual.

Os pacientes foram mantidos em unidade fechada, sob monitoração eletrocardiográfica, até o dia seguinte, quando, não havendo complicações, recebiam alta hospitalar. Foram administradas mais duas doses de cefalexina, por via oral, em intervalos de oito horas após o procedimento. Antes da alta, foi realizado ETT para avaliar a posição da prótese.

O seguimento foi realizado por meio de visitas ambulatoriais na semana seguinte e 30 dias, 90 dias e 180 dias após o implante. Foram realizados ETTs coincidindo com as datas das consultas médicas. Pelo menos um exame de eletrocardiografia dinâmica (Holter) de 24 horas foi realizado nos primeiros 6 meses após a alta. Os pacientes foram mantidos em uso de ácido acetilsalicílico, na dose de $5 \mathrm{mg} / \mathrm{kg}$ (ou $100 \mathrm{mg}$ em adultos) e orientados a realizar profilaxia antibiótica para endocardite infecciosa, por 6 meses.

As variáveis contínuas foram expressas como média e desvio padrão e as variáveis categóricas, como números e porcentagens. 

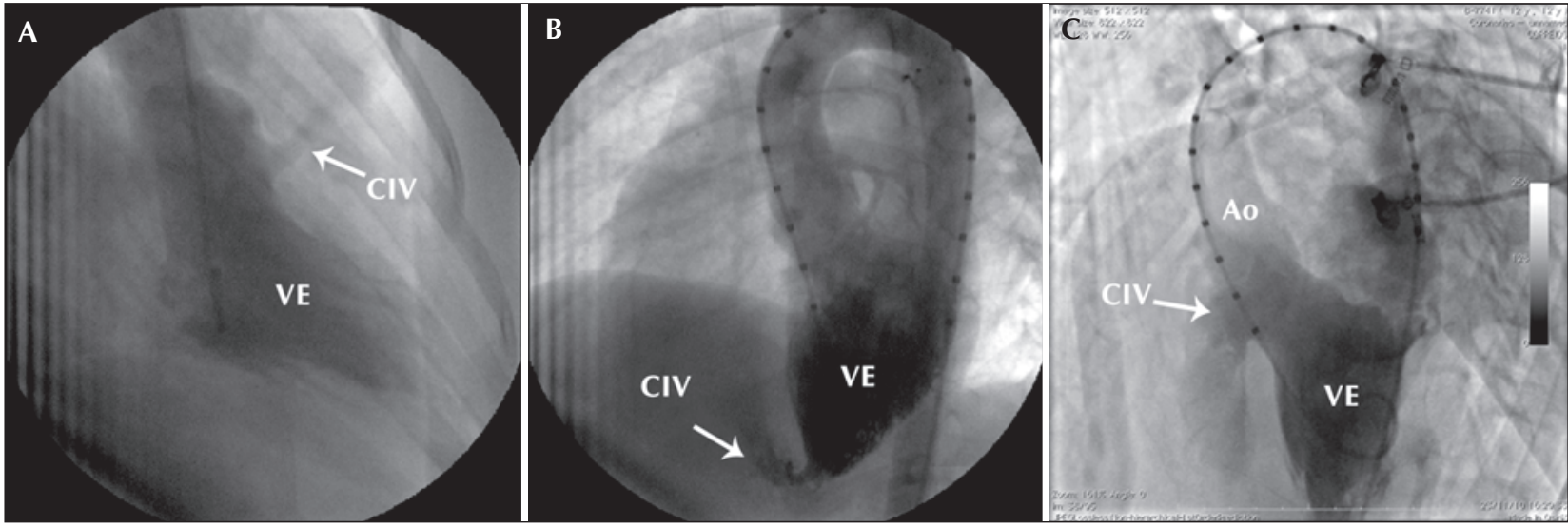

Figura 2 - Exemplos de ventriculografia esquerda para delinear os defeitos. Em A, comunicação interventricular muscular infundibular, tipicamente mais bem delineada em oblíqua anterior direita. Em B, comunicação interventricular muscular apical, em oblíqua anterior esquerda eixo longo. Em C, comunicação interventricular perimembranosa com aneurisma e duplo orifício, também em oblíqua anterior esquerda eixo longo. Ao = aorta; $\mathrm{CIV}=$ comunicação interventricular; $\mathrm{VE}=$ ventrículo esquerdo.

\section{RESULTADOS}

De março de 2010 a abril de 2011, 8 pacientes preencheram os critérios de inclusão e foram submetidos ao procedimento. Todos os pacientes eram assintomáticos, sendo 4 do sexo masculino, com idades que variaram de 7 anos a 32 anos (18,6 \pm 7,8 anos) e pesos, de $30 \mathrm{~kg}$ a $97 \mathrm{~kg}(66,12 \pm 24,7 \mathrm{~kg})$.

Entre os pacientes avaliados, 4 apresentaram CIV muscular (infundibular em 1, apical em 1 e trabecular em 2) e os restantes apresentaram CIV perimembranosa (Tabela). Entre esses pacientes, 3 apresentavam reduplicação de tecido tricúspide (aneurisma de septo membranoso) e 2 apresentavam duplo orifício. Os menores diâmetros dos defeitos variaram de $2 \mathrm{~mm}$ a $8,6 \mathrm{~mm}$ $(5 \pm 2,4 \mathrm{~mm})$.

O implante foi possível em todos os casos, sendo utilizadas 10 próteses nos 8 pacientes. A prótese mais utilizada foi a Lifetech Cera $^{\mathrm{TM}}$ tipo I; as próteses Amplatzer $^{\mathrm{TM}}$ VSD Muscular Occluder e Lifetech Cera ${ }^{\mathrm{TM}}$ tipo II também foram empregadas. O diâmetro das próteses utilizadas variou de $4 \mathrm{~mm}$ a $12 \mathrm{~mm}(7 \pm 2,8 \mathrm{~mm})$ e as bainhas, de $5 \mathrm{~F}$ a $7 \mathrm{~F}$.

O caso número 2 apresentava CIV muscular apical e foi submetido previamente a implante de uma prótese Amplatzer ${ }^{\text {TM }}$ Muscular VSD Occluder por via anterógrada, restando um orifício adicional, que foi ocluído dois anos depois, neste estudo. Foi utilizada uma segunda prótese Amplatzer ${ }^{\mathrm{TM}}$, do mesmo tamanho da anterior, implantada por via retrógrada.

As complicações do procedimento foram relacionadas à embolização de duas próteses, que foram retiradas utilizando-se cateteres especiais de resgate para corpo estranho. Oclusão imediata dos defeitos ocorreu em 6 casos $(75 \%)$, restando pequenos fluxos residuais em 2 pacientes.
O tempo de seguimento variou de 1 mês a 14 meses ( $7 \pm 5,5$ meses) e nenhum óbito ocorreu nesta casuística. Uma paciente apresentou episódio de bloqueio atrioventricular total (BAVT), com quadros sincopais na semana seguinte ao procedimento. Foi internada em unidade fechada, submetida a implante de marca-passo provisório e tratada com corticoterapia, obtendo reversão para ritmo sinusal com condução atrioventricular 1:1 no Holter de controle, assim permanecendo no acompanhamento tardio. Aos 6 meses, 7 pacientes $(87,5 \%)$ mostraram CIVs fechadas.

\section{DISCUSSÃO}

$\mathrm{Na}$ primeira descrição da técnica para o fechamento de CIV muscular, Lock et al. ${ }^{4}$ acreditavam que a trabeculação do ventrículo direito dificultaria o cruzamento do defeito pelo ventrículo direito e sugeriram a técnica utilizada até hoje. Nessa técnica, o defeito é cruzado pelo ventrículo esquerdo e uma guia de troca é laçada em algum lugar do coração direito (preferentemente a artéria pulmonar esquerda) e exteriorizada pelo acesso venoso, criando uma alça arteriovenosa. A bainha é introduzida pela extremidade venosa da guia e o implante da prótese é realizado do ventrículo esquerdo para o ventrículo direito. ${ }^{4}$ Com isso, conseguia-se excelente suporte da guia para trafegar bainhas calibrosas através dos defeitos.

Em 1994, Rigby e Redington ${ }^{5}$ descreveram pela primeira vez o fechamento de CIV perimembranosa com uso da dupla umbrella de Rashkind para persistência do canal arterial. Inicialmente tentavam abordar a CIV pelo ventrículo direito; se não tivessem sucesso, seguiam a rotina descrita por Lock et al. ${ }^{4}$.

O uso do oclusor Amplatzer ${ }^{\mathrm{TM}}$ de discos assimétricos para CIV perimembranosa impedia a utilização da via arterial para o implante ${ }^{19}$; dessa forma, a abordagem retrógrada para oclusão desse tipo de defeito não 

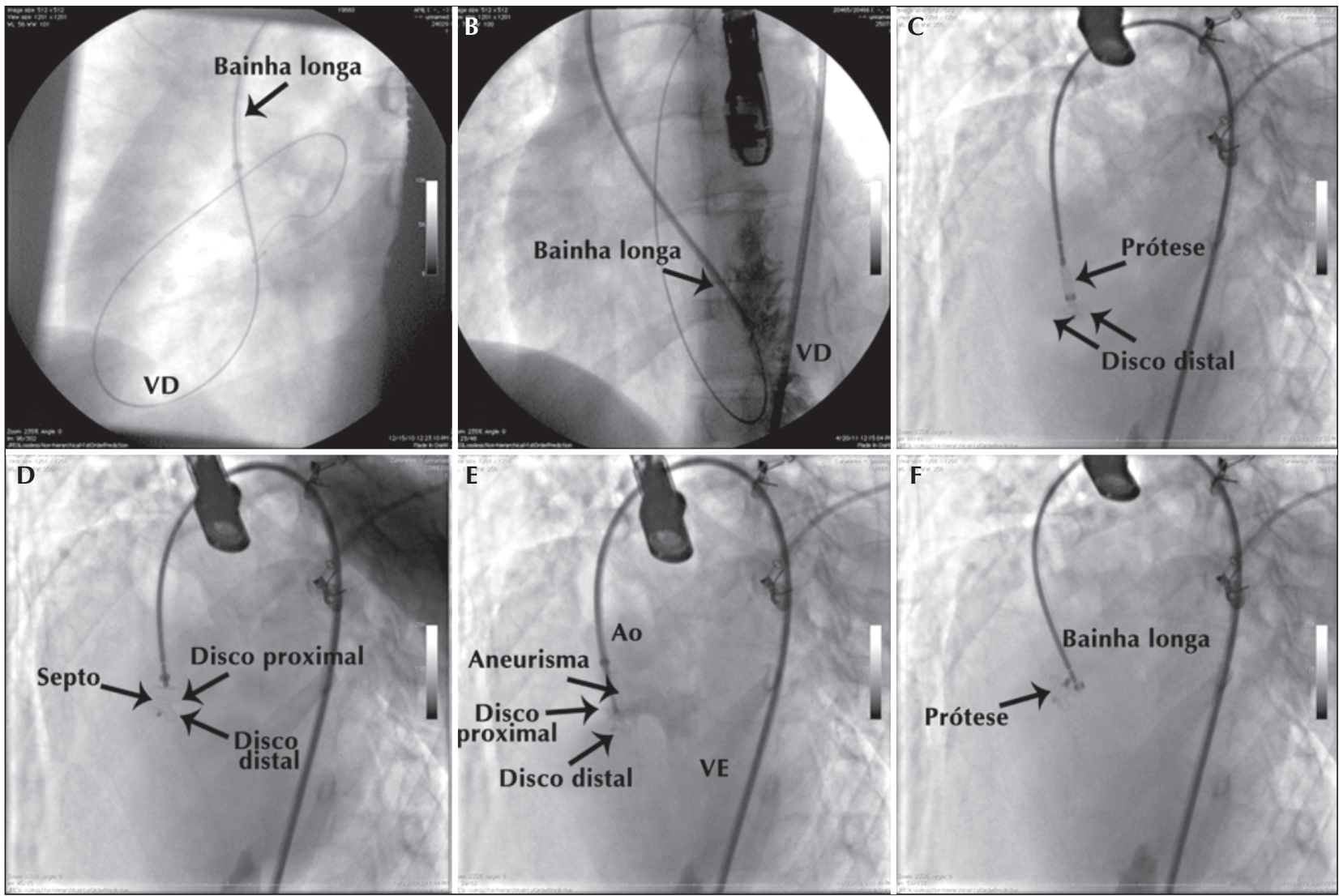

Figura 3 - Etapas do implante pela técnica retrógrada. Em A, guia no ramo esquerdo pulmonar depois de cruzada a comunicação interventricular pelo ventrículo esquerdo, com a bainha longa sendo introduzida sobre a guia. Em B, bainha longa posicionada em ventrículo direito, através de injeção pela válvula "Y". Em C, início do implante, com o disco distal exposto e tracionado em direção ao septo interventricular. Em D, disco proximal liberado no ventrículo direito. Em E, posição do dispositivo conferida por meio de nova injeção pela válvula "Y", sendo perfeitamente visualizada a posição adequada da prótese, com os discos dispostos de cada lado do septo interventricular. Em $F$, a prótese imediatamente depois de liberada. Ao = aorta; $V D=$ ventrículo direito; $V E$ = ventrículo esquerdo.

era tentada. O aparecimento dos dispositivos simétricos de duplo disco ${ }^{24,26}$ possibilitou o fechamento arterial das CIVs perimembranosas, embora essa variação técnica ainda não tivesse sido relatada.

A melhora do material auxiliar, como guias longas hidrofílicas e bainhas flexíveis e inquebráveis (non kinkable), fez crer que o fechamento das CIVs por via arterial poderia ser obtido, desde que se pudesse utilizar os dispositivos simétricos, de duplo disco. As vantagens dessa técnica eram óbvias. A primeira delas é a simplificação do procedimento. A realização da alça arteriovenosa na técnica tradicional é uma etapa delicada do implante. É muito importante que a guia seja retirada pela veia, sem cruzar o aparelho de sustentação tricuspídeo, a fim de se obter o trajeto mais retilíneo possível, permitindo a subida suave da bainha longa pela veia, até a aorta descendente, através da CIV. Ocasionalmente, o cruzamento do defeito tem que ser tentado mais de uma vez, até que se alcance esse objetivo. Da mesma forma, a captura da extremidade da guia, com o laço, pode ser difícil e acrescenta mais tempo ao procedimento. O procedimento necessita de operador com maior experiência em intervenção, além de bom conhecimento de anatomia.

Nitidamente, ao se eliminar essa etapa, o tempo de procedimento é reduzido e, com isso, paciente e equipe médica são menos expostos à radiação. Uma das desvantagens da abordagem retrógrada é não ter o cateter arterial em ventrículo esquerdo para monitorar o posicionamento do dispositivo no septo. Isso pode ser facilmente superado por meio de injeções pela bainha através de uma válvula em "Y" (Touhey-Borst), dispensando o cateter arterial, que, ainda assim, poderá ser utilizado por meio de punção na artéria femoral contralateral.

Outro pequeno problema encontrado durante a realização dos procedimentos foi a posição mais verticalizada da bainha longa, o que acarreta certo grau de tensão na prótese, que fica posicionada quase em ângulo reto em relação ao eixo do sistema de entrega. Isso causa alguma dificuldade para visualizar a relação do disco distal com o septo e faz com que exista o risco da passagem inadvertida do disco distal para o ventrí- 


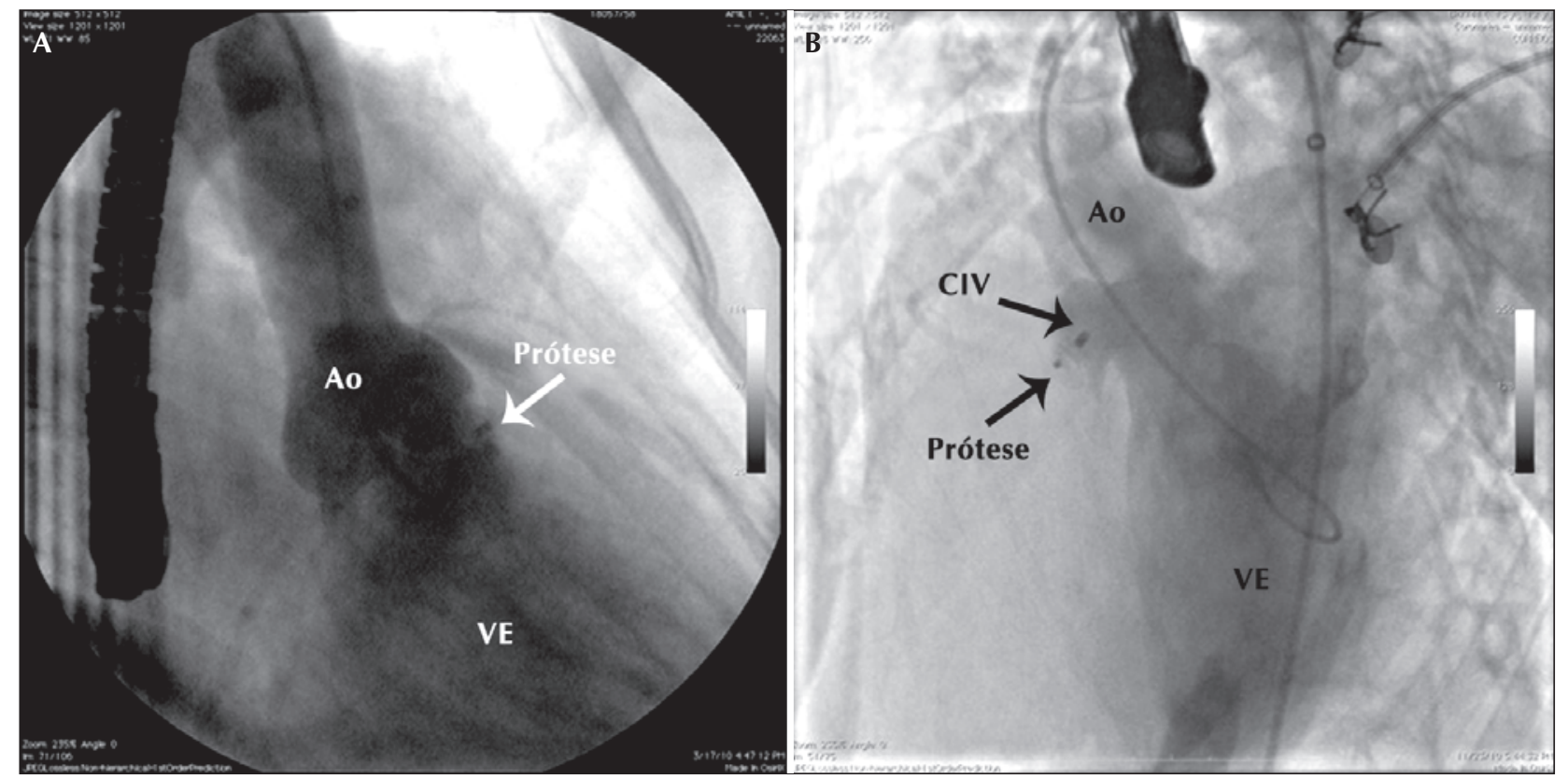

Figura 4 - Angiografias de controle demonstrando, à esquerda, comunicação interventricular muscular infundibular e, à direita, comunicação interventricular perimembranosa, ambas completamente fechadas com os dispositivos. Note-se que, no painel da direita, os dois orifícios foram ocluídos com uma única prótese. Ao = aorta; CIV = comunicação interventricular; VE = ventrículo esquerdo.

TABELA

Caracterização da população do estudo

\begin{tabular}{|c|c|c|c|c|c|c|c|}
\hline Identificação & Sexo & $\begin{array}{l}\text { Idade } \\
\text { (anos) }\end{array}$ & $\begin{array}{c}\text { Tipo } \\
\text { morfológico }\end{array}$ & $\begin{array}{l}\text { Entrada do } \\
\text { VE (mm) }\end{array}$ & $\begin{array}{l}\text { Saída do } \\
\text { VD (mm) }\end{array}$ & Dispositivo & $\begin{array}{l}\text { Diâmetro } \\
\text { (mm) }\end{array}$ \\
\hline $\begin{array}{l}\text { Paciente } 1 \text { - } \\
\text { BFGF }\end{array}$ & $\mathrm{F}$ & 32 & $\begin{array}{l}\text { Muscular } \\
\text { infundibular }\end{array}$ & 7 & 3,5 & $\begin{array}{c}\text { Cera }^{\mathrm{TM}} \text { VSD } \\
\text { tipo I }\end{array}$ & 6 \\
\hline $\begin{array}{l}\text { Paciente } 2 \text { - } \\
\text { BPS }\end{array}$ & $M$ & 7 & Muscular apical & 10 & $3+2$ & $\begin{array}{l}\text { AGA VSD } \\
\text { musc }\end{array}$ & $4+4$ \\
\hline \multirow[t]{2}{*}{$\begin{array}{l}\text { Paciente } 3 \text { - } \\
\text { AMB }\end{array}$} & M & 16 & Perimembranosa & 7 & 4 & $\begin{array}{c}\text { Cera }^{\mathrm{TM}} \text { VSD } \\
\text { tipo II }\end{array}$ & 7 \\
\hline & & & & & & $\begin{array}{c}\text { Cera }^{\text {TM }} \text { VSD } \\
\text { tipo I }\end{array}$ & 7 \\
\hline $\begin{array}{l}\text { Paciente } 4 \text { - } \\
\text { LSDP }\end{array}$ & $\mathrm{F}$ & 12 & Perimembranosa & 14 & $7+1$ & $\begin{array}{c}\text { Cera }^{\mathrm{TM}} \text { VSD } \\
\text { tipo I }\end{array}$ & 8 \\
\hline $\begin{array}{l}\text { Paciente } 5 \text { - } \\
\text { EJS }\end{array}$ & M & 17 & $\begin{array}{l}\text { Muscular } \\
\text { trabecular }\end{array}$ & 6 & 3 & $\begin{array}{c}\text { Cera }^{\mathrm{TM}} \text { VSD } \\
\text { tipo I }\end{array}$ & 4 \\
\hline $\begin{array}{l}\text { Paciente } 6 \text { - } \\
\text { VBA }\end{array}$ & $\mathrm{F}$ & 17 & $\begin{array}{l}\text { Muscular } \\
\text { trabecular }\end{array}$ & 9,1 & 8,6 & $\begin{array}{c}\text { Cera }^{\mathrm{TM}} \text { VSD } \\
\text { tipo I }\end{array}$ & 10 \\
\hline $\begin{array}{l}\text { Paciente } 7 \text { - } \\
\text { LBB }\end{array}$ & $\mathrm{F}$ & 22 & Perimembranosa & 5 & 4 & $\begin{array}{c}\text { Cera }^{\mathrm{TM}} \text { VSD } \\
\text { tipo I }\end{array}$ & 5 \\
\hline \multirow[t]{2}{*}{$\begin{array}{l}\text { Paciente } 8 \text { - } \\
\text { RAS }\end{array}$} & M & 26 & Perimembranosa & 8 & $3+8$ & $\begin{array}{c}\text { Cera }^{\text {TM }} \text { VSD } \\
\text { tipo I }\end{array}$ & 5 \\
\hline & & & & & & $\begin{array}{c}\text { Cera }^{\text {TM }} \text { VSD } \\
\text { tipo I }\end{array}$ & 12 \\
\hline
\end{tabular}

$\mathrm{F}=$ feminino; $\mathrm{M}=$ masculino; musc = muscular; $\mathrm{VD}=$ ventrículo direito; $\mathrm{VE}=$ ventrículo esquerdo.

culo esquerdo. Outra preocupação é que, pela possibilidade de tensão nas bordas do orifício da CIV, possam surgir distúrbios de condução ou mesmo BAVT. A impressão dos autores é que o nível de tensão 

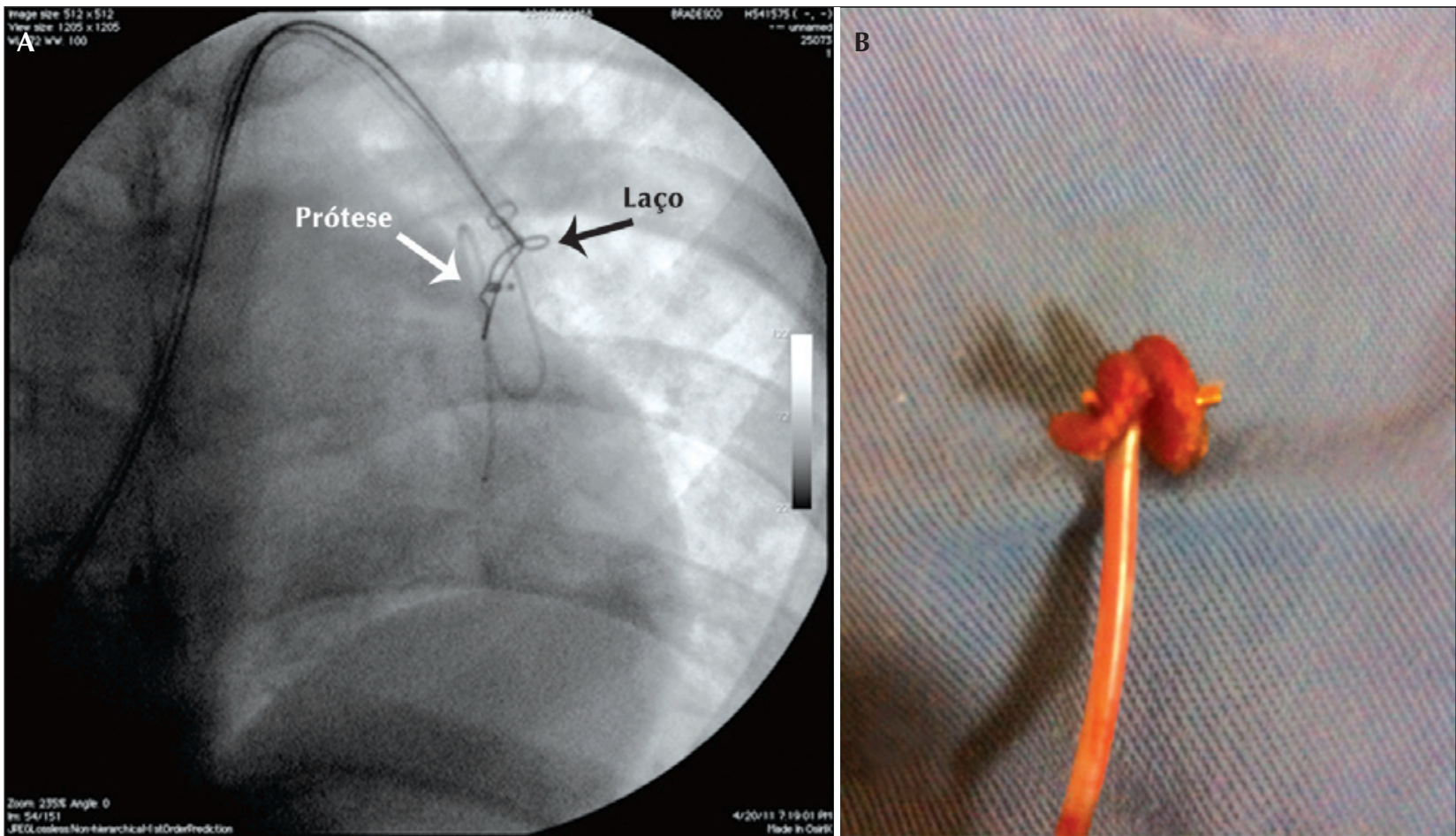

Figura 5 - Detalhes da captura de uma prótese embolizada. Em A, dispositivo sendo laçado em ramo esquerdo pulmonar. Em B, dispositivo retirado, ainda preso pelo laço.

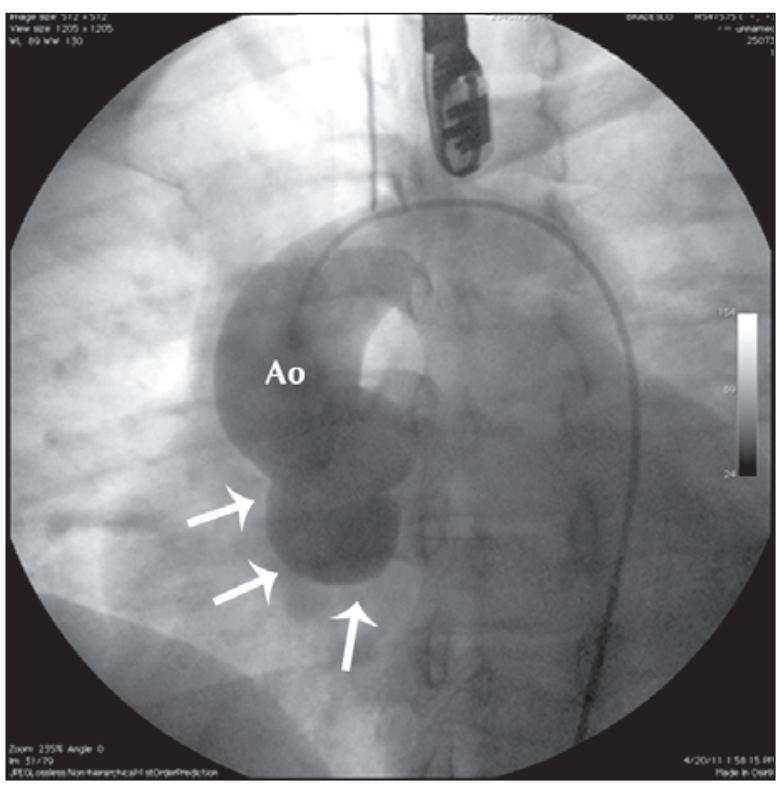

Figura 6 - Aortografia panorâmica do caso número 8 (RAS). Observa-se valva aórtica tricúspide e competente com grande dilatação do seio de Valsalva não-coronário, que dificultou a visualização adequada das comunicações interventriculares do paciente. Ao $=$ aorta.

exercido pela bainha não deve ser maior que o que ocorre durante a técnica tradicional e, nesta pequena casuística, não houve nenhum caso de bloqueio ou distúrbio de condução durante o procedimento, nem isso se constituiu em dificuldade maior ou fator impeditivo para a realização do implante.

Na maioria dos casos, foi utilizada guia hidrofílica 0,032 polegada/260 cm para cruzar o defeito, que era posicionada, preferentemente, na artéria pulmonar esquerda, para dar estabilidade à introdução da bainha. Apenas para efeito de experimentação, a bainha longa foi introduzida sobre a guia e posicionada em veias cavas e, mesmo, no interior do ventrículo direito, sem maiores dificuldades ou problemas. Nas poucas vezes em que foi necessário mais suporte da guia, o cateter JR era avançado sobre ela e posicionado na artéria pulmonar, e a guia hidrofílica era substituída por uma guia super stiff 0,035 polegada/260 cm. Isso ocorreu em 2 casos, por ocasião da introdução dos dispositivos de maior tamanho. A posição mais vertical da guia em ventrículo direito favorece a passagem mais suave da bainha longa e, na maioria das vezes, torna desnecessária a substituição da guia.

Deve-se salientar o fato de que a realização da variação retrógrada em crianças pequenas, com amplas CIVs, pode obrigar a introdução de bainhas calibrosas, acima de $7 \mathrm{~F}$, em artérias de pequeno diâmetro. Nesses casos, a via anterógrada, tradicional, deverá ser utilizada.

Neste estudo procurou-se testar essa variação técnica nos diversos tipos morfológicos de CIV e ela foi possível em todos, mesmo nas CIVs musculares apicais, em que tradicionalmente se introduz a bainha por via 
jugular. A passagem da bainha longa foi obtida com extrema facilidade em todos os casos.

Ocorreram duas embolizações de dispositivo, nenhuma delas relacionada à técnica utilizada. Na primeira embolização, foi implantada uma prótese Lifetech Cera $^{\text {TM }}$ tipo II para CIV perimembranosa com aneurisma, por via anterógrada. A prótese foi liberada com ambos os discos, inadvertidamente, no ventrículo esquerdo, migrando para a aorta descendente, de onde foi facilmente resgatada com cateter laço (Figura 5). Em seguida foi implantada uma prótese Lifetech Cera ${ }^{\mathrm{TM}}$ tipo I, de mesmo diâmetro, por via retrógrada, com sucesso. No segundo caso, também CIV perimembranosa com aneurisma e duplo orifício, havia grande dilatação do seio não-coronário de Valsalva, sem regurgitação aórtica (Figura 6), e essa imagem era superposta à da CIV, dificultando, sobremaneira, sua correta visualização. Foram implantadas duas próteses Lifetech Cera $^{T M}$ tipo I por via retrógrada. A menor se deslocou durante o implante da maior e embolizou para o território pulmonar, de onde foi resgatada. Aparentemente a prótese maior conseguiu ocluir os dois orifícios, que eram contíguos, ao ecocardiograma de controle imediato pós-procedimento. Esse paciente ainda não realizou ecocardiograma de seguimento de um mês.

Fluxos residuais periprotéticos imediatos aconteceram em dois casos com CIV perimembranosa. Os autores acreditam que os fluxos residuais decorreram mais do tipo de defeito envolvido que, propriamente, da técnica de implante.

A paciente que apresentou BAVT era portadora de CIV muscular trabecular, não muito próxima da valva aórtica, e que, portanto, não apresentava risco maior para distúrbio de condução. O procedimento transcorreu sem eventos e o traçado eletrocardiográfico manteve-se inalterado em todo o período de monitoração durante a internação. Os autores acreditam que, também nesse caso, a arritmia não decorreu do tipo de técnica empregada.

\section{CONCLUSÕES}

Apesar do pequeno número de casos, foi demonstrado que a utilização da via retrógrada para a oclusão percutânea das CIVs simplifica a realização do procedimento. Essa via mostrou-se factível e eficaz em todos os tipos morfológicos de CIV em que foi executada, uma vez que seja possível o uso de próteses, simétricas, de duplo disco. É uma técnica facilmente reproduzível. Os autores sugerem essa técnica como opção para a oclusão percutânea das CIVs de qualquer morfologia.

\section{CONFLITO DE INTERESSES}

Os autores declaram não haver conflito de interesses relacionado a este manuscrito.

\section{REFERÊNCIAS}

1. Tynan M, Anderson RH. Ventricular septal defect. In: Anderson RH, Becker EA, MacCartney FJ, editors. Pediatric cardiology. $2^{\text {nd }}$ ed. New York: Churchill Livingstone; 2002. p. 983-1014.

2. Backer CL, Winters RC, Zales VR, Takami H, Muster AJ, Benson DW Jr, et al. Restrictive ventricular septal defect: how small is too small to close? Ann Thorac Surg. 1993;56(5):1014-9.

3. Mavroudis C, Backer CL, Idrees FS. Ventricular septal defect. In: Mavroudis C, Baker CL, editors. Pediatric cardiac surgery. 2nd ed. St. Louis, MO: Mosby; 1994. p. 201-24.

4. Lock JE, Block PC, McKay RG, Baim DS, Keane JF. Transcatheter closure of ventricular septal defects. Circulation. 1988; 78(2):361-8.

5. Rigby $\mathrm{ML}$, Redington $\mathrm{AN}$. Primary transcatheter umbrella closure of perimembranous ventricular septal defects. $\mathrm{Br}$ Heart J. 1994;72(4):368-71.

6. Sideris EB, Walsh KP, Haddad JL, Chen CR, Ren SG, Kulkarni $\mathrm{H}$. Occlusion of congenital ventricular septal defects by buttoned device. "Buttoned device" Clinical Trials International Register. Heart. 1997;77(3):276-9.

7. Latiff HA, Alwi M, Kandhavel G, Samion H, Zambahari R. Transcatheter closure of multiple muscular ventricular septal defects using Gianturco coils. Ann Thorac Surg. 1999;68(4): 1400-1.

8. Amin Z, Gu X, Berry JM, Bass J, Titus JL, Urness M, et al. New device for closure of muscular ventricular septal defects in a canine model. Circulation. 1999;100(3):320-8.

9. Hijazi ZM, Hakim F, Al-Fadley F, Abdelhamid J, Cao Q-L. Transcatheter closure of single muscular ventricular septal defects using the Amplatzer muscular VSD occluder: initial results and technical considerations. Catheter Cardiovasc Interv. 2000;49(2):167-72.

10. Arora R, Trehan V, Kumar A, Kalra GS, Nigam M. Transcatheter closure of congenital ventricular septal defects: experience with various devices. J Interv Cardiol. 2003;16(1):83-91.

11. Thanopoulos BD, Karanassios E, Tsaousis G, Papadopoulos GS, Stefanadis C. Catheter closure of congenital/acquired muscular VSDs and perimembranous VSDs using the Amplatzer devices. J Interv Cardiol. 2003;16(5):399-407.

12. Arora R, Trehan V, Thakur AK, Mehta V, Sengupta PP, Nigam M. Transcatheter closure of congenital muscular ventricular septal defect. J Interv Cardiol. 2004;17(2):109-15.

13. Holzer R, Balzer D, Cao QL, Lock K, Hijazi ZM; Amplatzer Muscular Ventricular Septal Defect Investigators. Device closure of muscular ventricular septal defects using the Amplatzer muscular ventricular septal occluder: immediate and midterm results of a U.S. registry. J Am Coll Cardiol. 2004; 43(7):1257-63.

14. Chessa M, Carminati M, Cao QL, Butera G, Giusti S, Bini RM, et al. Transcatheter closure of congenital and acquired muscular ventricular septal defects using the Amplatzer device. J Invasive Cardiol. 2002;14(6):322-7.

15. Michel-Behnke I, Le TP, Waldecker B, Akintuerk $H$, Valeske $\mathrm{K}$, Schranz D. Percutaneous closure of congenital and acquired ventricular septal defects-considerations on selection of the occlusion device. J Interv Cardiol. 2005;18(2):89-99.

16. Thanopoulos BD, Rigby ML. Outcome of transcatheter closure of muscular ventricular septal defects with Amplatzer ventricular septal occluder. Heart. 2005;91(4):513-6.

17. Jameel AA, Arfi AM, Arif H, Amjad K, Omar GM. Retrograde approach for device closure of muscular ventricular septal defects in children and adolescents, using the Amplatzer muscular ventricular septal defect occluder. Pediatr Cardiol. 2006;27(6):720-8.

18. Bass JL, Kalra GS, Arora R, Masura J, Gavora P, Thanopoulos $B D$, et al. Initial human experience with the Amplatzer 
perimembranous ventricular septal occluder device. Catheter Cardiovasc Interv. 2003;58(2):238-45.

19. Hijazi ZM, Hakim F, Haweleh AA, Madani A, Tarawna W, Hiari $A$, et al. Catheter closure of perimembranous ventricular septal defects using the new Amplatzer membranous VSD occluder: initial clinical experience. Catheter Cardiovasc Interv. 2002;56(4):508-15.

20. Thanopoulos BD, Tsaousis GS, Karanasios E, Elephterakis NG, Paphitis C. Transcatheter closure of perimembranous ventricular septal defects with the Amplatzer asymmetric ventricular septal defect occluder: preliminary experience in children. Heart. 2003;89(8):918-22.

21. Pedra CAC, Pedra SRF, Esteves CA, Pontes SC Jr, Braga SL, Arrieta SR, et al. Percutaneous closure of perimembranous ventricular septal defects with the Amplatzer device: technical and morphological considerations. Catheter Cardiovasc Interv. 2004;61(3):403-10
22. Queiroz FJ, Rossi Filho RI, Ramos S, Esteves C, Queiróz DS, Machado PR, et al. Oclusão percutânea das comunicações interventriculares. Experiência inicial. Arq Bras Cardiol. 2005. 85(3):174-9.

23. Carminati M, Butera G, Chessa M, Drago M, Negura D, Piazza L. Transcatheter closure of congenital ventricular septal defect with Amplatzer septal occluders. Am J Cardiol. 2005; 96(12A):52L-58L.

24. Zuo J, Xie J, Yi W, Yang J, Zhang J, Li J, et al. Results of transcatheter closure of perimembranous ventricular septal defect. Am J Cardiol. 2010;106(7):1034-7.

25. Gu M, Bai Y, Zhao X, Zheng X, Li W, Qin YW. Transcatheter closure of postoperative residual perimembranous ventricular septal defects. Ann Thorac Surg. 2009;88(5):1551-5.

26. Gu M, You X, Zhao X, Zheng X, Qin YW. Transcatheter device closure of intracristal ventricular septal defects. Am J Cardiol. $2011 ; 107(1): 110-3$ 